\title{
COMPORTAMENTO DE CULTIVARES DE TRITICALE E DE TRIGO NO VALE DO PARANAPANEMA (1)
}

JOÃO CARLOS FELICIO (2) e ANTONIO WILSON PENTEADO FERREIRA FILHO, Seção de Arroz e Cereais de Inverno, Instituto Agronomico, e BENEDITO DE CAMARGO BARROS, Secão de Doensas dis Plantas Alimenticias Básicas $e$ Olerícolas, Instituto Biológico.

\section{RESUMO}

Foram instalados nos anos de 1979,1980 e 1981, na Fazenda Santa Inês, no Vale do Paranapanema, município de Maracai, ensaios comparativos entre quinze cultivares de triticale e três de trigo cultivados no Estado de São Paulo. Considerando os ensaios em conjunto, não houve diferença estatística, para a produção de grãos, entre todos os cultivares estudados; houve, porém, uma tendência de o 'TCEP 77138' (triticale) apresentar maior média de produtividade. Os cultivares de triticale apresentaram maior resistência a Puccinia graminis iritici, em condições de campo, quando comparados aos de trigo, que demonstraram reações de suscetibilidade. No entanto, foram mais sensiveis às manchas foliares causadas por Helminthosporium sp. e Septoria sp., e mostraram grãos mais enrugados, embora 'TCEP 77138' apresentasse grãos com boa formação, isto $\tilde{e}$, com muito pouco enrugamento.

\section{INTRODUÇÃO}

O triticale, cultivo novo ( $(\boldsymbol{7})$ e relativamente desconhecido, é produto de cruzamento entre espécies dos gêneros Triticum (ao qual pertence o trigo) e Secale (ao qual pertence o centeio).

(1) Recebido para publicação a 30 de abril de 1982.

(2) Com bolsa de suplementação do CNPq. 
Um dos seus problemas (9) era a forma anormal do endosperma. Em lugar de uma semente dura e vítrea, típica do trigo (Triticum durum L.), a semente madura do triticale carecia de brilho e ajresentava uma casca enrugada (3). Como resultado prático, o grão tornava-se inatrativo para o agricultor, e a semente enrugada refletia un peso hectolitrico baixo (peso por unidade de volume). A semente malformada constituía um ambiente deficiente para o embrião, proporcionando baixa germinação.

Os melhores cultivares de triticale apresentam pesos hectolitricos de 72 a $76 \mathrm{~kg} / \mathrm{hl}$; entretanto, para os melhores trigos, esse índice é superior a $80 \mathrm{~kg} / \mathrm{hl}$.

Um avanço significativo (9) para o melhoramento do triticale foi logrado com a obtenção de uma linha de triticale hexaplóide, altamente fértil, denominado de "Armadillo", que possui uma combinação de características, única nos triticales: alta fertilidade, maior peso hectolítrico, melhor rendimento, insensibilidade ao fotoperiodo, precocidade e um gene de nanismo.

As ferrugens (Puccinia sp.) dos cereais são consideradas a ameaça mais séria para a produção de triticale (5). Acredita-se que o trigo seja resistente às ferrugens que atacam o centeio, e que este não seja atacado pelas ferrugens específicas do trigo. Em conseqüência, é de esperar que o triticale, que combina o complemento cromossômico completo do trigo e centeio, poderia ser selecionado para resistência a ambas as formas de ferrugem.

Ensaios conduzidos em 1973 (1), comparando o rendimento de trigo e triticale demonstraram boa produtividade de alguns triticales e resistência às ferrugens, mas com a característica de enrugamento dos grãos.

Os cultivares de triticale apresentavam maior tolerância ao $\mathrm{Al}^{3+}$, em solução nutritiva, porém mostraram maior sensibilidade em relação aos de centeio, nas mesmas condiçōes, demonstrando que parte da tolerância ao $\mathrm{Al}^{3+}$ foi transferida pelo centeio (2).

Trabalhos com triticale realizados nos Cerrados do Brasil central (8) demonstraram comportamento semelhante ao trigo e rendimentos superiores 25 a $30 \%$ aos cultivares de trigo semeados na região.

O objetivo do trabalho foi estudar o comportamento de cultivares de triticale ('Iriticum secale) comparado a cultivares comerciais de trigo (Triticum aestivum) na regiāo do Vałe do Paranapanema.

\section{MATERIAL E Mêtodos}

O delineamento estatístico empregado foi blocos ar acaso, com quatro repetições, sendo os experimentos conduzidos durante os anos de 1979, 1980 e 1981, na Fazenda Santa Inês, no município de Maracaí.

Foram utilizados dezoito tratamentos, quinze de diferentes triticales e três dos cultivares de trigo IAC-5, CNT-9 e IAC-17. 
Entre os cultivares de triticale, enumerados no quadro 1, sete procederam do Centro Nacional de Pesquisa de Trigo (PFT) e oito do Centro de Pesquisa da FECOTRIGO (CEP e TCEP).

QUADRO 1. Relação dos tratamentos, com especificação do «pedigree» de cultivares de triticale e de trigo utilizados no presente trabalho

\begin{tabular}{|c|c|c|c|}
\hline \multicolumn{3}{|c|}{ Tratamento - Cultivar } & Cruzamento/Pedigree \\
\hline 1. & CEP 75709 & $=$ & FS $1897-1 B$ \\
\hline 2. & CEP 76287 & $=$ & $\mathbf{M}_{1} \mathrm{~A}$ \\
\hline 3. & TCEP 77136 & $=$ & $\begin{array}{l}M_{2}^{A-C M L}=X 8386-D-2 y-O M-100 y-102 B-105 y-1-1 M-100 x- \\
6 M-O y-O A\end{array}$ \\
\hline 4. & TCEP 77137 & $=$ & $\begin{array}{l}M_{2} \mathrm{~A}-\mathrm{CML}=\mathrm{X} 8386-\mathrm{D}-2 \mathrm{y}-\mathrm{OM}-100 \mathrm{y}-103 \mathrm{~B}-109 \mathrm{y}-1 \mathrm{y}-1 \mathrm{M}- \\
100 \mathrm{y}-\mathrm{OA}\end{array}$ \\
\hline 5. & TCEP 77138 & $=$ & $\mathbf{M}_{2} \mathrm{~A}-\mathrm{CML}=\mathrm{X} 8386-\mathrm{D}-2 \mathrm{y}-\mathrm{AM}-100 \mathrm{y}-103 \mathrm{~B}-109 \mathrm{y}-1 \mathrm{M}-100 \mathrm{y}-\mathrm{OA}$ \\
\hline 6. & TCEP 77.139 & $=$ & $\begin{array}{l}M_{\mathrm{O}}^{2} \mathrm{~A}-\mathrm{CML}=\mathrm{X} 8386-\mathrm{D}-2 \mathrm{y}-\mathrm{AM}-100 \mathrm{y}-103 \mathrm{~B}-109 \mathrm{y}-1 \mathrm{Y}-1 \mathrm{M}-101 \mathrm{y}- \\
\mathrm{OA}\end{array}$ \\
\hline 7. & TCEP 77140 & $=$ & $\mathbf{M}_{2} \mathrm{~A}-\mathrm{CML}=\mathrm{X} 8386$ \\
\hline 8. & TCEP 77142 & $=$ & Chapala - snoopy X-M $\mathrm{A}$ \\
\hline 9. & $\mathrm{PF}^{* T} 763$ & $=$ & $\mathrm{M}_{2} \mathrm{~A}-\mathrm{CML}=\mathrm{X} 8386-\mathrm{D}-2 \mathrm{y}-\mathrm{OM}-100 \mathrm{y}-102 \mathrm{~B}-101 \mathrm{y}-\mathrm{Oy}$ \\
\hline 10. & PFT 764 & $=$ & $\mathrm{M}^{2} \mathrm{~A}-\mathrm{CML}=\mathrm{X} 8386-\mathrm{D}-2 \mathrm{y}-\mathrm{OM}-101 \mathrm{y}-101 \mathrm{~B}-101 \mathrm{y}-\mathrm{Oy}$ \\
\hline 11. & PET 765 & $=-$ & $\mathrm{M}_{2}^{2} \mathrm{~A}-\mathrm{CML}=\mathrm{X} 8386-\mathrm{D}-2 \mathrm{y}-\mathrm{OM}-101 \mathrm{y}-103 \mathrm{~B}-102 \mathrm{y}-\mathrm{Oy}$ \\
\hline 12. & PFT 766 & $=$ & $\mathrm{M}_{2}^{\prime} \mathrm{A}-\mathrm{CML}=\mathrm{X} 8386-\mathrm{D}-2 \mathrm{y}-\mathrm{OM}-101 \mathrm{y}-101 \mathrm{~B}-107 \mathrm{y}-\mathrm{Oy}$ \\
\hline 13. & PET 768 & $=-$ & $\mathrm{CIN}-\mathrm{M}_{2} \mathrm{~A}^{2}=\mathrm{X} 15401-100 \mathrm{y}-\mathrm{Oy}$ \\
\hline 14. & PFI 7622 & $=$ & $\mathbf{M}_{2} \mathrm{~A}-\mathrm{CML}=\mathrm{X} 8386-\mathrm{D}-2 \mathrm{y}-\mathrm{OM}-100 \mathrm{y}-103 \mathrm{~B}-100 \mathrm{y}-\mathrm{Oy}$ \\
\hline 15. & PFT 7651 & $=$ & F-4-UM-2M-2y-3C-1y \\
\hline 16. & LAC-5 & $==$ & FN - Kenya 58 × PG1 \\
\hline 17. & CNT-9 & $=$ & IAS-46 $\times$ IAS-49 x Tobari 66 \\
\hline 18. & IAC -17 & $=$ & IAS-20 $\times$ IRN-526-63 \\
\hline
\end{tabular}

Antes da semeadura, foram retiradas amostras compostas dos solos dos locais estudados, cujos resultados analíticos se encontram no quadro 2 .

QUADRO 2. Análise química dos solos onde foram executados os experimentos na Fazenda Santa Inês - Maracaí

Determinações a

Anos

\begin{tabular}{|c|c|c|c|}
\hline $\mathrm{pH}$ int & 5,7 & 5,5 & 5,9 \\
\hline 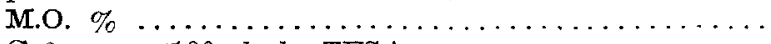 & 3,4 & 2,9 & 2,9 \\
\hline $\mathrm{Ca}^{2} *$ e.mg $/ 100 \mathrm{mI}$ de TFSA $\ldots \ldots \ldots \ldots \ldots \ldots$ & 6,4 & 3,0 & 3,6 \\
\hline $\mathbf{M g} \div$ e.mg/100mi de TFSA $\ldots \ldots \ldots \ldots \ldots$ & 1,7 & 1,2 & $\mathbf{1 , 6}$ \\
\hline $\mathrm{PO}^{3}-\mu \mathrm{mg} / \mathrm{ml}$ de TFSA $\ldots \ldots \ldots \ldots$ & 16,0 & 8,0 & 11,0 \\
\hline $\mathrm{K}+\mu \mathrm{mg} / \mathrm{ml}$ de TFSA $\ldots \ldots \ldots \ldots$ & 268 & 232 & 132 \\
\hline $\mathrm{Al}^{3}+$ e.mg $/ 100 \mathrm{ml}$ de TFSA $\ldots$. & 0,0 & 0,0 & 0,0 \\
\hline
\end{tabular}

(a) Análise efetuada na Seção de Fertilidade do Solo, Instituto Agronômico, Campinas. 
Nos três anos de experimentação, os ensaios foram semeados durante a segunda quinzena de abril.

Cada ensaio foi constituído de 72 parcelas, com cinco linhas de $5 \mathrm{~m}$ de comprimento, espaçadas de $0,20 \mathrm{~m}$, com um espaçamento lateral entre as parcelas de $0,60 \mathrm{~m}$.

A semeadura foi feita na base de 80 sementes viáveis por metro de sulco, equivalendo a 400 sementes por metro quadrado, e por ocasião da colheita foram colhidas as plantas das cinco linhas, com uma área útil, portanto, de $5 \mathrm{~m}^{2}$.

A adubação utilizada, aplicada a lanço, de acordo com a análise do solo (Quadro 2) compunha-se de $30 \mathrm{~kg}$ de $\mathrm{N}$, 90kg de $\mathrm{P}_{2} \mathrm{O}_{3}$ e $20 \mathrm{~kg}$ de $\mathrm{K}_{2} \mathrm{O}$, por hectare, nas formas respectivas de sulfato de amônio com $20 \%$ de $\mathrm{N}$, superfosfato simples com $20 \%$ de $\mathrm{P}_{2} \mathrm{O}_{5}$, e cloreto de potássio com $60 \%$ de $\mathrm{K}_{2} \mathrm{O}$.

Para avaliar o comportamento dos cultivares em relação às principais doenças em condições naturais de infecção, foram empregadas escalas de avaliação de sintomas, em condições de campo. As avaliações foram feitas em planta adulta, examinando-se os colmos e as folhas superiores de cerca de vinte plantas por parcela. Para a ferrugem-do-colmo (Puccinia graminis Pers. forma sp. tritici Ericks et Henn) e ferrugem-da-folha (Puccinia recondita Rob. ex. Desm. forma sp. tritici Ericks), empregou-se a escala modificada de Cobb, contida em MEHTA (6), que é composta por um número que estima a porcentagem de área de tecido atacada pelos fungos, no colmo e na folha, acrescido de uma letra simbolizando o tipo de reação: $\mathrm{S}=$ suscetivel (uredossoro grande, coalescente sem necrose e sem clorose); MS $=$ moderadamente suscetivel (uredossoro médio); $M=$ intermediário (diversos tipos de reação); $\mathrm{MR}=$ moderadamente resistente (uredossoro pequeno); e $\mathrm{R}=$ resistente (uredossoro minúsculo rodeado de áreas necróticas).

Para o complexo septoriose mais helmintosporiose (Septoria sp. e Helminthosporium sp.) nas folhas, foi estimada a porcentagem da área foliar infectada, observando-se as quatro folhas superiores das plantas.

A altura das plantas de cada cultivar foi medida no campo, sendo uma amostragem de cada parcela, levando em consideração a distância do nível do solo ao ápice da espiga, mantendo-se as plantas esticadas; para avaliação do enrugamento dos grãos foram compuíadas notas de 1 a 5 por julgamento visual, representando 1 o maior enrugamento do endosperma e 5, a superfície do endosperma lisa semelhante à do trigo, acrescidas dos sinais + ou - para determinar as aproximaçōes.

Na determinação do ciclo dos cultivares estudados, foi considerado o número de dias da emergência da planta até sua maturação completa, adotando-se como ciclo precoce, maturaçāo até 120 dias, ciclo médio de 121 a 135 dias, e tardio de 136 dias oú mais. 


\section{RESULTADOS E DISCUSSÃO}

A germinação nos três experimentos foi considerada boa para os cultivares de trigo e triticale.

Os dados de produtividade de grãos, em quilograma por hectare, dos materiais estudados no triênio 1979-81, encontram-se no quadro 3 . A análise conjunta dos experimentos não apresentou diferença significativa para produtividade de grãos $(F=0,933)$ entre os cultivares de trigo e de triticale estudados no periodo. Houve efeito altamente significativo $(\mathrm{F}=2,66)$ para a interação anos $\mathrm{x}$ tratamentos e para anos $(F=74,62)$. Somente em 1981 é que se observaram diferenças significativas entre os cultivares de triticale.

QUADRO 3. Produtividade de grãos de cultivares de triticale $\mathrm{e}$ trigo nos anos de 1979-81 na Fazenda Santa Inês, município de Maracaí

\begin{tabular}{|c|c|c|c|c|c|}
\hline & \multirow{2}{*}{ Cultivar } & \multicolumn{3}{|c|}{ Produção } & \multirow{2}{*}{ Média } \\
\hline & & 1979 & 1980 & 1981 & \\
\hline & & & ـ & - & + \\
\hline \multirow[t]{2}{*}{ CEP } & 75709 & 1.380 & 1.775 & 258 & 1.138 \\
\hline & 76287 & 1.373 & 2.165 & 810 & 1.449 \\
\hline \multirow[t]{6}{*}{ TCEP } & 77136 & 1.213 & 1.390 & 1.050 & 1.218 \\
\hline & 137 & 1.358 & 2.165 & 1.195 & 1.573 \\
\hline & 138 & 1.433 & 2.278 & 1.338 & 1.683 \\
\hline & 139 & 1.048 & 2.510 & 1.210 & 1.589 \\
\hline & 140 & 1.348 & 2.173 & 1.158 & 1.559 \\
\hline & 142 & 1.283 & 1.760 & 1.223 & 1.422 \\
\hline \multirow[t]{7}{*}{$\mathrm{PFT}$} & 763 & 1.288 & 2.070 & 1.128 & 1.495 \\
\hline & 764 & 1.105 & 2.188 & 1.140 & 1.478 \\
\hline & 765 & 1.065 & 1.785 & 1.223 & 1.358 \\
\hline & 766 & 1.275 & 2.465 & 1.168 & 1.636 \\
\hline & 768 & 1.515 & 2.135 & 958 & 1.536 \\
\hline & 7622 & 1.530 & 1.955 & 1.175 & $1.5 \overline{5} 3$ \\
\hline & 7651. & 1.643 & 1.733 & 858 & 1.411 \\
\hline \multicolumn{2}{|c|}{ IAC-5-Trigo } & 1.053 & 2.125 & 895 & 1.358 \\
\hline \multicolumn{2}{|c|}{ CNT-9-Trigo } & 1.298 & 1.970 & 808 & 1.358 \\
\hline \multicolumn{2}{|c|}{ IAC-17-Trigo } & 1.358 & 2.170 & 1.165 & 1.564 \\
\hline \multirow{3}{*}{\multicolumn{2}{|c|}{$\begin{array}{l}\text { F. } \ldots \ldots \ldots \ldots \\
\text { d.m.s. (Tukey } 5 \%) \\
\text { C.V. }(\%) \quad \ldots \ldots \ldots\end{array}$}} & $1,63 \mathrm{NS}$ & $1,56 \mathrm{NS}$ & $8,96 * \%$ & $0,933 \mathrm{NS}$ \\
\hline & & 878 & 1.468 & ธ59 & 788 \\
\hline & & 20,09 & 21,49 & 16,08 & \\
\hline
\end{tabular}


Apesar de os resultados alcançados não mostrarem điferenças significativas entre os tratamentos, pode-se notar uma tendência para maior produção de grãos em quilograma/hectare do cultivar de triticale TCEP-77138 e do cultivar de trigo IAC-17.

A maioria dos cultivares de triticale apresentou menor índice de infecção de Puccinia graminis tritici (ferrugem-do-colmo), como é demonstrado no quadro 4, comparado aos cultivares de trigo que apresentaram reações de suscetibilidade, embora a intensidade da dcença tenha sido baixa. Para a Puccinia recondita (ferrugem-da-folha), a resistência dos cultivares de triticale foi bem maior se comparada às notas obtidas pelos cultivares de trigo IAC-5 e IAC-17, suscetíveis à moléstia.

Para as manchas foliares (Helminthosporium sp. e Septoria sp.), aparentemente, observou-se certa inversão de valores, $\epsilon \mathrm{m}$ relação às ferrugens, isto é, os cultivares de triticale apresentaram raior suscetibilidade quando comparados aos de trigo, que, apesar de não apresentarem resistência, foram mais tolerantes às moléstias.

Comparando o porte das plantas (quadro 4), verifica-se que os cultivares de triticale são, de modo geral, mais baixos, o que poderia proporcionar maior resistência ao acamamento.

Analisando o quadro 5, verifica-se que os cultivares de trigo apresentaram peso hectolítrico mais elevado. Destacaram-se como os melhores cultivares de triticale: TCEP-77138, TCEP-77139, TCEP-77140, PFT-763 e PFT-766, que apresentaram melhor estabilidade para peso hectolitrico e o peso de mil sementes no biênio estudado.

O peso em gramas de mil sementes, correlacionado com o número de sementes por grama, resultou no valor de $-0,796$ para o ano de 1980 e de $-0,819$ para o de 1981, valores esses que se aproximam do limite de significância ao nivel de $0,1 \%$. Para as demais correlaçóes estudadas, como peso hectolítrico $\mathrm{x}$ peso de mil sementes e peso hectolítrico $x$ número de sementes por grama, não houve resultados estatisticamente significativos.

Os cultivares de triticale apresentaram, quanto à característica de enrugamento, melhor aspecto que os estudados por CAMARGO \& FELiCIO (1), notadamente os TCEP-77138, TCEP-77139 e PFT-766, com nota $4+$, somente sendo superados nos dois anos de estudo pelo cultivar de trigo IAC-17, com nota 5. 


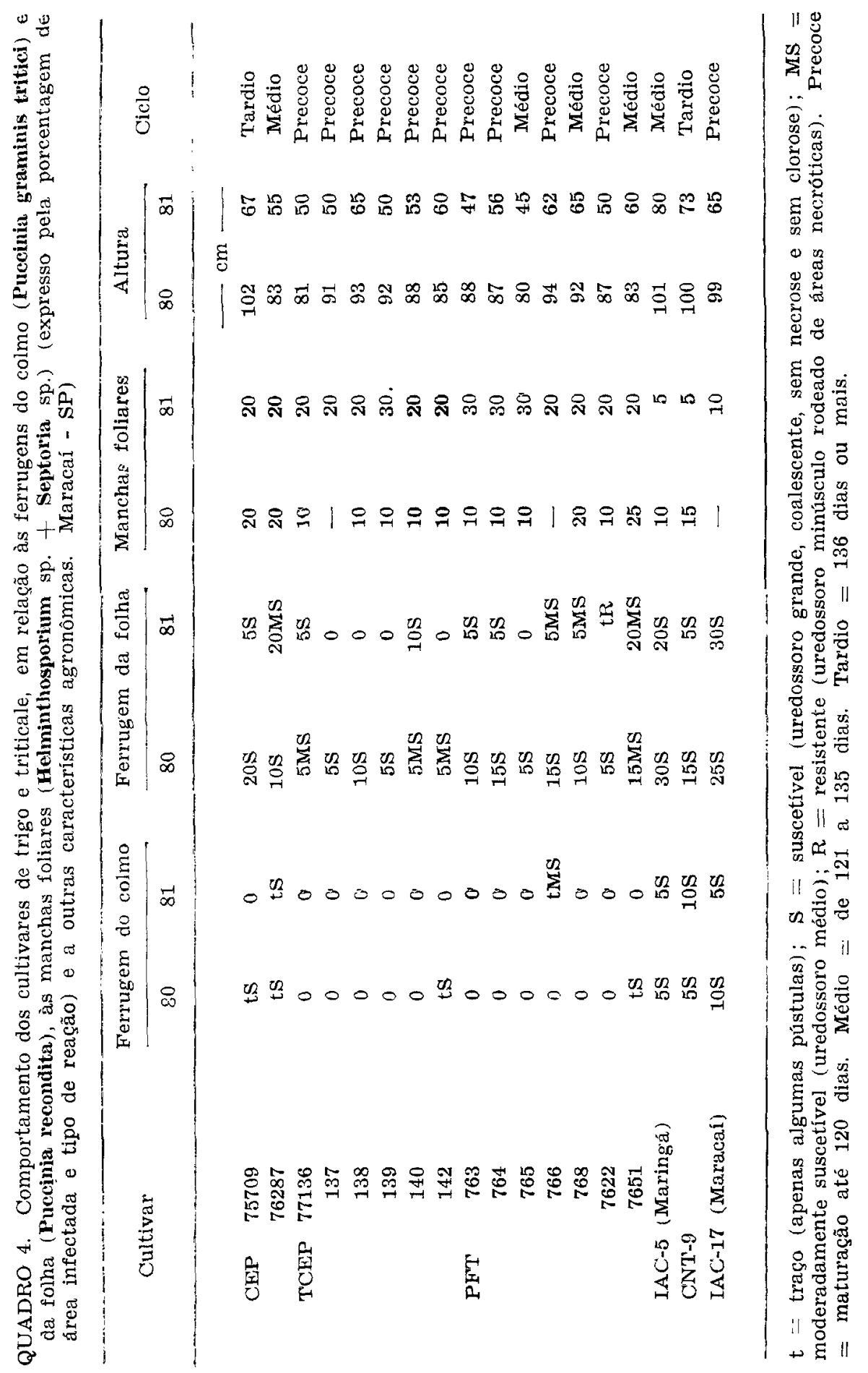




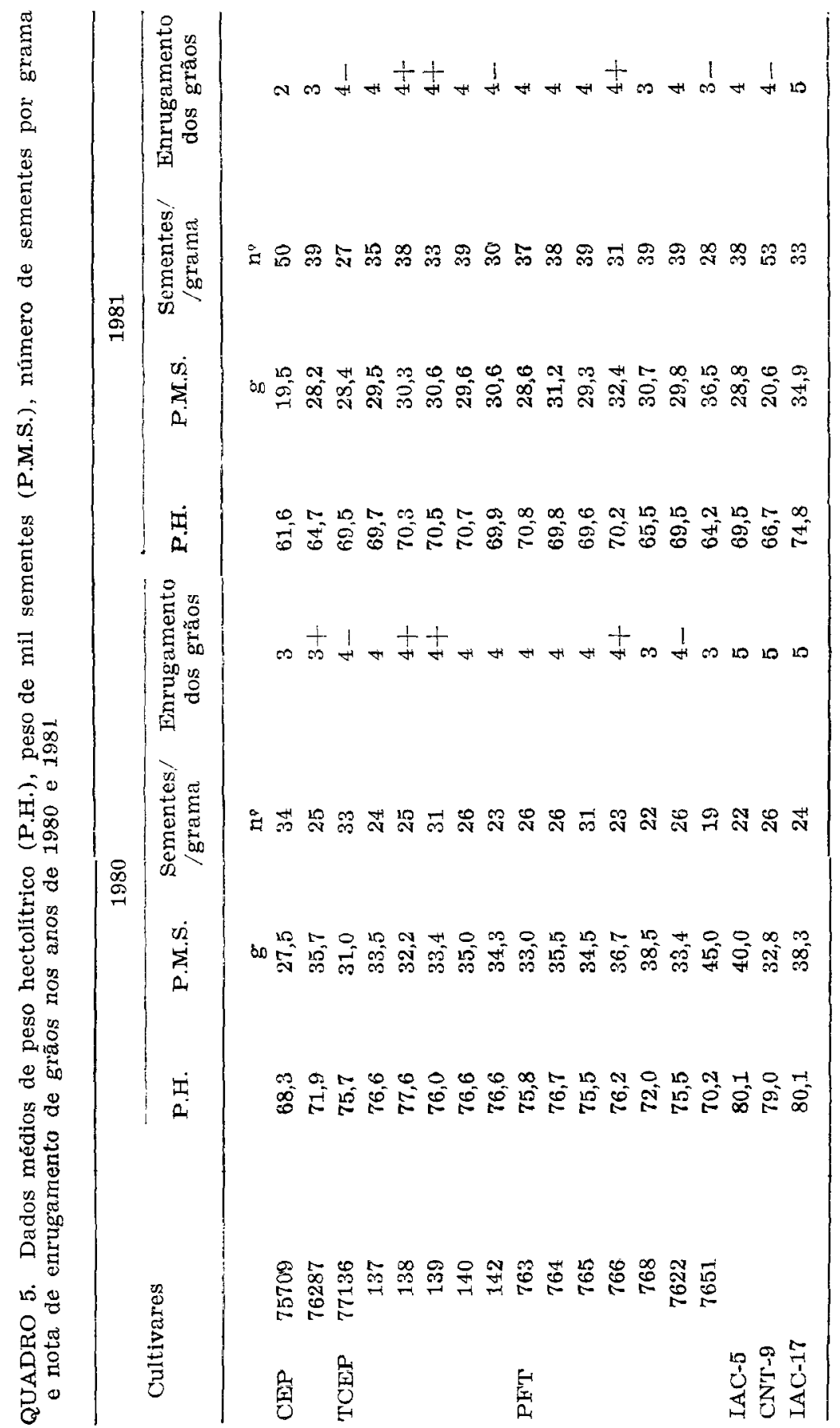




\section{CONCLUSÕES}

Os resultados obtidos no presente trabalho permitem tirar as seguintes conclusōes:

1. Em média, não houve diferenças significativas quanto à produção de grãos entre os cultivares de triticale e trigo estudados.

2. O cultivar de triticale TCEP-77138 destacou-se dos demais pela produção de grãos, resistência às ferrugens, melhor aspecto do endosperma e alto peso hectolítrico.

3. Os cultivares de triticale foram mais sensiveis a manchas foliares devidas ao Helminthosporium sp. e Septoria sp. do que os cultivares de trigo.

\section{SUMMARY}

\section{BEHAVIOUR OF TRITICALE AND WHEAT CULTIVARS IN THE} PARANAPANEMA VALLEY, STATE OF SĀO PAULO, BRAZIL

Fifteen triticale and three wheat cultivars were evaluated in three experimental trials carried out at the Santa Inês Farm, Maracaí, in the Paranapanema Valley during the years of 1979,1980 and 1981 . The wheat and triticale cultivars did no differ in relation to grain yield, however, the triticale TCEP-77138 presented high yield associated with resistance to stem rust, and with high weight of one hectoliter of seeds. Triticale cultivars were more sensitive than wheat to leaf diseases caused by Helminthosporium sp. and Septoria sp.

\section{REFERENCIAS BIBLIOGRAFICAS}

1. CAMARGO, C.E.O. \& FELICIO, J.C. Estudo comparativo preliminar entre cultivares de triticale e um cultivar de trigo no Estado de São Paulo. Bragantia, Campinas, 34:287-293, 1975.

2. -__ \& _ _ . Tolerância de cultivares de trigo, triticale e centeio em diferentes níveis de alumínio em solução nutritiva. Bragantia, Campinas, $43(1): 9-15,1984$.

3. CENTRO INTERNACIONAL DE MEJORAMIENTO DE MAIZ Y TRIGO CIMMYT. Revisão dos Programas do CIMMYT. Ell Batan, México, 1978. 141p.

4. GOMES, F.P. Curso de estatística experimental. Piracicaba, Escola Superior de Agricultura «Luiz de Queiroz», 1963. 384p.

5. LOPES BENITEZ, A. Identificación de las formas de Puccinia graminis Pers. que atacan al triticale. Chapingo, MÉxico, Colégio de Post Graduados, Escuela Nacional de Agricultura, 1971. 60p. Tesis. (M.S.)

6. MEHTA, Y.R. Doenças do trigo e seu controle. São Paulo, Ceres, 1978. 190p.

7. RILEY, R. \& CHAPMAN, V. The comparison of wheat-rye wheat Aegilops amplidiploidis. Journal Agricultural Science, 49:246-250, 1957.

8. SILVA, A.R.; ANDRADE, J.M.V. de; LEITE, J.C. Comportamento de triticale nos cerrados do Distrito Federal e em várzeas na Região de Curvelo, Minas Gerais. In: REUNLÃO DA COMISSÃO NORTE-BRASILEIRA DE PESQUISA DE TRIGO, 7., Ponta Grossa, PR, 1981. 28p. (Mimeografado)

9. ZILLINSKY, F. \& BORLAUG, N.E. Progress in developing triticale as an economic crop. México, CIMMYT, 1971. 27p. (Research Bulletin, 17) 